Pacific Journal of Mathematics

ANOTHER CHARACTERIZATION OF AE(0)-SPACES 


\title{
ANOTHER CHARACTERIZATION OF AE(0)-SPACES
}

\author{
VesKo M. VALOV
}

\begin{abstract}
We prove that a space $X$ is an absolute extensor for the class of all zero-dimensional spaces if and only if $X$ is an upper semi-continuous compact-valued retract of a power of the real line.
\end{abstract}

1. Introduction. Dugundji spaces were introduced by Pelczynski [5]. Later Haydon [4] proved that the class of Dugundji spaces coincides with the class of all compact absolute extensors for zero-dimensional compact spaces (briefly, AE(0)). After Haydon's paper, compact $\mathrm{AE}(0)$ spaces have been extensively studied (see Ščepin's review [9]); let us note the following result of Dranishnikov [3]: a compact $X$ is an $\mathrm{AE}(0)$-space if and only if for every embedding of $X$ in a Tychonoff cube $I^{\tau}$ there exists an upper semi-continuous compact-valued (br. usco) mapping $r$ from $I^{\tau}$ to $X$ such that $r(x)=\{x\}$, for each $x \in X$ (such a usco mapping will be called a usco retraction).

Chigogidze [2] extended the notion of $\mathrm{AE}(0)$ from the class of compact spaces to that of completely regular spaces and gave a characterization of such $\mathrm{AE}(0)$-spaces.

The aim of the present paper is to give another characterization of completely regular $\mathrm{AE}(0)$-spaces which is similar to the above mentioned result of Dranishnikov. We prove that $X \in \mathrm{AE}(0)$ iff $X$ is a usco retract of $R^{\tau}$ for some $\tau$, where $R$ is the real line with the usual topology. Our technique is different from Dranishnikov's.

The author is indebted to S. Nedev and M. Ganster for useful discussions.

2. Notations and terminology. All spaces considered are completely regular and all single-valued mappings are continuous. A set-valued mapping $r$ from $X$ to $Y$ is called upper semi-continuous (br. u.s.c.) if the set $r^{\#}(U)=\{x \in X: r(x) \subset U\}$ is open in $X$ whenever $U$ is open in $Y$. We say that a usco mapping $r$ is minimal if every usco selection for $r$ coincides with $r$. It follows from the Kuratowski-Zorn lemma that every usco mapping has a minimal usco selection.

A mapping $f$ from $Y$ to $X$, where $Y \subset Z$, is called $Z$-normal if, for every continuous function $g$ on $X$, the function $g \circ f$ is continuously extendable to $Z$. A space $X$ is called an absolute extensor for zero-dimensional spaces [2], if every $Z$-normal mapping $f$ from $Y$ to $X$, where $Y \subset Z$ 
and $\operatorname{dim} Z=0$, is continuously extendable to $Z$; if $f$ is continuously extendable only to a neighbourhood of $Y$ in $Z$, the space $X$ is called an absolute neighbourhood extensor for 0-dimensional space, briefly ANE(0). Here, dim stands for the dimension defined by finite functionally open covers.

A mapping $f$ from $X$ to $Y$ will be called 0-soft [2], if for every 0 -dimensional space $Z$ and every two $Z$-normal mappings $g: Z_{0} \rightarrow X, h$ : $Z_{1} \rightarrow Y$ with $Z_{0} \subset Z_{1} \subset Z$ and $f \circ g=h \mid Z_{0}$, there exists a $Z$-normal mapping $k: Z_{1} \rightarrow X$ such that $g=k \mid Z_{0}$ and $f \circ k=h$. In the case $Z$ is paracompact and $Z_{0}$ and $Z_{1}$ are closed subsets of $Z$, one gets Ščepin's notion [8] of a 0 -soft mapping, defined earlier.

A space $X$ is said to be a multivalued absolute (resp. neighbourhood) extensor (br. $X \in \mathrm{MA}(\mathrm{N}) \mathrm{E}$ ) if every $Z$-normal mapping $f: Z_{0} \rightarrow X$ with $Z_{0} \subset Z$, can be extended to a usco mapping from $Z$ (resp. from a neighbourhood of $Z_{0}$ in $Z$ ) to $X$.

A mapping $f: X \rightarrow Y$ is said to be functionally open if $f(U)$ is functionally open in $Y$ for every functionally open subset $U$ of $X$.

Let $A$ be a subset of $X$. We dentoe by $G_{\delta}(A)$ the $G_{\delta}$-closure of $A$ in $X$; i.e. the set $\left\{x \in X\right.$ : every $G_{\delta}$-subset of $X$ containing $x$ intersects $\left.A\right\}$. Finally, let $X=\Pi\left\{X_{s}: s \in S\right\}$ and $B \subset S$. Then $p_{B}$ stands for the natural projection from $X$ onto $X_{B}=\Pi\left\{X_{s}: s \in B\right\}$. If $U$ is a subset of $X$, then $k(U)$ denotes the family $\left\{B: p_{B}^{-1}\left(p_{B}(U)\right)=U\right\}$.

\section{3. $\mathrm{AE}(0)$-spaces.}

LEMMA 1. Let $X=\Pi\left\{X_{s}: s \in S\right\}$ be a product of separable metric spaces and let $U$ be $a G_{\delta}$-set in $X$. Then there exists a countable set $B \subset S$ such that $p_{B}(U)$ is a $G_{\delta}$-set in $X_{B}$ and $G_{\delta}(U)=X_{S \backslash B} \times p_{B}(U)$. If $U$ is open in $X$ then $G_{\delta}(U)$ is functionally open in $X$.

Proof. Put $M=X \backslash G_{\delta}(U)$. By a result of R. Pol and E. Pol [6] there exists a countable set $B \subset S$ such that $p_{B}(U)$ is a $G_{\delta}$-set in $X_{B}$ and $p_{B}(U) \cap p_{B}(M)=\varnothing$. Hence $p_{B}^{-1}\left(p_{B}(U)\right) \cap M=\varnothing$. Since $p_{B}\left(G_{\delta}(U)\right)$ $=p_{B}(U)$, we have $B \in k\left(G_{\delta}(U)\right)$, so $G_{\delta}(U)=p_{B}(U) \times X_{S \backslash B}$. If $U$ is open in $X$ then $p_{B}(U)$ is functionally open in $X_{B}$. Thus, $G_{\delta}(U)$ is functionally open too.

The proof of the follwing (actually known) lemma is an easy exercise on the definition of a minimal usco mapping.

LEMMA 2. Let $r$ be a minimal usco mapping from $X$ to $Y$ and let $U$ be an open set in $Y$. Then the following holds:

(i) $r(x) \subset \operatorname{cl}(U)$ for every $x \in \operatorname{Int}\left(\operatorname{cl}\left(r^{\#}(U)\right)\right)$; 
(ii) $\operatorname{cl}\left(r^{-1}(U)\right)=\operatorname{cl}\left(r^{\#}(U)\right)$, where $r^{-1}(U)=\{x \in X: \quad r(x) \cap U \neq$ $\varnothing\}$.

Let $Y=\Pi\left\{Y_{s}: s \in S\right\}$ be a product of separable metric spaces and let $X \subset Y$. Let $r$ be a u.s.c. mapping from $Y$ to $X$. A subset $B$ of $S$ is called $r$-admissible if $B \in k\left(\operatorname{cl}\left(r^{\#}(U \cap X)\right)\right)$ for every standard open subset $U$ of $Y$ with $B \in k(U)$. The above definition is a simple modification of the definition of $e$-admissible set, given by Shirokov [11]. The following lemma was actually proved by Shirokov [11].

LeMma 3. Let $Y=\Pi\left\{Y_{s}: s \in S\right\}$ be a product of separable metric spaces, $X \subset Y$ and let $r$ be a u.s.c. mapping from $Y$ to $X$. Then we have:

(i) for every set $B \subset S$ there is a r-admissible set $A$ containing $B$ and card $A=\operatorname{card} B$;

(ii) a union of r-admissible subsets of $S$ is $r$-admissible too.

LeMma 4. Let $Y=\Pi\left\{Y_{s}: s \in S\right\}$ be a product of separable metric spaces, $X \subset Y$ and let $r$ be a minimal usco mapping from $Y$ to $X$. Suppose $B$ is a r-admissible subset of $S$. Then the following conditions are fulfilled:

(i) $B \in k\left(\operatorname{cl}\left(r^{\#}\left(\bigcup_{i=1}^{n} U_{i} \cap X\right)\right)\right)$ for every finite family $\left\{U_{i}: i=\right.$ $1, \ldots, n\}$ of standard open subsets of $Y$ with $B \in \bigcap_{i=1}^{n} k\left(U_{i}\right)$;

(ii) $p_{B}(r(x))=p_{B}(r(y))$ whenever $p_{B}(x)=p_{B}(y)$.

Proof. (i) Let $U=\bigcup_{i=1}^{n} U_{i}$. By Lemma 2(ii) we have

$$
\begin{aligned}
\operatorname{cl}\left(r^{\#}(U \cap X)\right) & =\operatorname{cl}\left(r^{-1}(U \cap X)\right)=\operatorname{cl}\left(\bigcup_{i=1}^{n} r^{-1}\left(U_{i} \cap X\right)\right) \\
& =\bigcup_{i=1}^{n} \operatorname{cl}\left(r^{-1}\left(U_{i} \cap X\right)\right)=\bigcup_{i=1}^{n} \operatorname{cl}\left(r^{\#}\left(U_{i} \cap X\right)\right) .
\end{aligned}
$$

Since $B$ is $r$-admissible, $B \in k\left(\operatorname{cl}\left(r^{\#}\left(U_{i} \cap X\right)\right)\right)$ for each $i$. Thus, $B \in$ $k\left(\operatorname{cl}\left(r^{\#}(U \cap X)\right)\right)$.

(ii) Let $p_{B}(x)=p_{B}(y)$ and $p_{B}(r(y)) \subset p_{B}(V)$, where $V$ is open in $Y$. Since $r(y)$ is compact, $V$ can be considered as a finite union $\bigcup_{i=1}^{n} V_{i}$ of standard open subsets of $Y$ with $B \in \bigcap_{i=1}^{n} k\left(V_{i}\right)$. Then, by (i), we have $B \in k\left(\operatorname{cl}\left(r^{\#}(V \cap X)\right)\right)$. Consequently, $B \in k\left(\operatorname{Int}\left(\operatorname{cl}\left(r^{\#}(V \cap X)\right)\right)\right)$. Thus, $x \in \operatorname{Int}\left(\operatorname{cl}\left(r^{\#}(V \cap X)\right)\right)$ because $y \in r^{\#}(V \cap X)$. Hence, by Lemma 2(i), $r(x) \subset \operatorname{cl}(V \cap X)$ i.e. $p_{B}(r(x)) \subset \operatorname{cl}\left(p_{B}(V)\right)$. The last inclusion shows that $p_{B}(r(x)) \subset p_{B}(r(y))$. Analogously, $p_{B}(r(y)) \subset p_{B}(r(x))$. Therefore $p_{B}(r(x))=p_{B}(r(y))$. 
A mapping $f: X \rightarrow Y$ is said to have a polish kernel [2], if there exists a polish (i.e. complete separable metric) space $P$ such that $X$ is $C$-embedded in $Y \times P$ and $f$ coincides with the restriction $p_{Y} \mid X$, where $p_{Y}$ : $Y \times P \rightarrow Y$ is the natural projection. The following lemma is proved by Chigogidze [2].

Lemma 5. Let the mapping $f$ from $X$ to $Y$ have a polish kernel, where $X$ and $Y$ are $\mathrm{AE}(0)$-spaces. Then $f$ is 0 -soft if and only if $f$ is functionally open.

LEMMA 6. Let $Y=\Pi\left\{Y_{s}: s \in S\right\}$ be a product of separable metric spaces and let $r$ be a minimal usco retraction from $Y$ to $X$. Then for every $r$-admissible set $B \subset S$ the following conditions are fulfilled:

(i) the restriction $p_{B} \mid X$ is functionally open;

(ii) $p_{B}(X)$ is a usco retract of $Y_{B}$.

Proof. (i) First we prove that for every $\mathrm{C} \subset \mathrm{S}$ the projection $\mathrm{p}_{\mathrm{C}}$ is functionally open. Let $U$ be a functionally open subset of $Y$. Then, by Lemma 1, there exists a countable set $D \subset S$ such that $U=p_{D}^{-1}\left(p_{D}(U)\right)$. This permits us to present $U$ as a countable union $\bigcup_{i=1}^{\infty} U_{i}$ of standard open subsets of $Y$ with $D \in k\left(U_{i}\right)$, for each $i$. Hence, $p_{C}(U)=$ $\bigcup_{i=1}^{\infty} p_{C}\left(U_{i}\right)$. Since every $p_{C}\left(U_{l}\right)$ is a standard open subset of $Y_{C}$, the set $p_{C}(U)$ is a countable union of functionally open subsets of $Y_{C}$. Therefore $p_{C}(U)$ is functionally open.

Now, suppose $B$ is $r$-admissible and $U$ is functionally open in $X$. Since $G_{\delta}\left(r^{\#}(U)\right)$ is functionally open in $Y$ (by Lemam 1), in order to prove that $p_{B} \mid X$ is functionally open it suffices to show that $p_{B}(U)=$ $p_{B}\left(G_{\delta}\left(r^{\#}(U)\right)\right) \cap p_{B}(X)$. Let $x \in X$ and let $p_{B}(x)=p_{B}(y)$ for some $y \in G_{\delta}\left(r^{\#}(U)\right)$. If we assume $r(y) \subset X \backslash U$ then $y \in r^{\#}(X \backslash U)$. However $r^{\#}(X \backslash U)$ is a $G_{\delta}$-set in $Y$ because $X \backslash U$ is a zero-set in $X$. Hence, $r^{\#}(X \backslash U) \cap r^{\#}(U) \neq \varnothing$, which is impossible. Thus, $r(y) \cap U \neq \varnothing$. By Lemma 4(ii), we have $p_{B}(x)=p_{B}(r(x))=p_{B}(r(y))$, so $p_{B}(x) \in p_{B}(U)$. Therefore $p_{B}\left(G_{\delta}\left(r^{\#}(U)\right)\right) \cap p_{B}(X) \subset p_{B}(U)$. The inverse inclusion is obvious.

(ii) Let $B$ be a $r$-admissible set. Define a compact-valued mapping $r_{1}$ : $Y_{B} \rightarrow p_{B}(X)$ by letting $r_{1}\left(p_{B}(x)\right)=p_{B}(r(x))$. Lemma 4(ii) implies that this definition is correct and that $r_{1}\left(p_{B}(x)\right)=p_{B}(x)$ for every $x \in X$. It remains to prove that $r_{1}$ is u.s.c. Let $r_{1}\left(p_{B}\left(x_{0}\right)\right) \subset U$ for some $x_{0} \in Y$, where $U$ is open in $Y_{B}$. Then, by Lemma 4(i), we have $B \in$ $k\left(\operatorname{cl}\left(r^{\#}\left(p_{B}^{-1}(U) \cap X\right)\right)\right)$. Consequently, $B \in k(V)$, where $V=$ $\operatorname{Int}\left(\operatorname{cl}\left(r^{\#}\left(p_{B}^{-1}(U) \cap X\right)\right)\right)$. The set $p_{B}(V)$ is a neighbourhood of $p_{B}\left(x_{0}\right)$ 
because $x_{0} \in r^{\#}\left(p_{B}^{-1}(U) \cap X\right)$. Let $p_{B}(x) \in p_{B}(V)$. Then $x \in V$ and, by Lemma 2(i), $r(x) \subset \operatorname{cl}\left(p_{B}^{-1}(U) \cap X\right)$; so $r_{1}\left(p_{B}(x)\right) \subset \operatorname{cl}(U)$. Therefore, $r_{1}$ is u.s.c.

LEMMA 7. Let $Y=\Pi\left\{Y_{s}: s \in S\right\}$ be a product of separable metric spaces and let $X$ be a usco retract of $Y$. Then the following conditions are fulfilled:

(i) $X$ is $C$-embedded in $Y$;

(ii) there exists a set $B \subset S$ of cardinality $w(X)$ such that $p_{B} \mid X$ is a homeomorphism and $p_{B}(X)$ is a usco retract of $Y_{B}$.

Proof. (i) Suppose $f$ is a continuous function on $X$. Consider the family $\mathscr{L}$ of all open intervals in $R$ with rational endpoints. Using Lemma 1 , for every $U \in \mathscr{L}$ choose a countable set $B(U) \subset S$ such that $B(U) \in$ $k\left(G_{\delta}\left(r^{\#}\left(f^{-1}(U)\right)\right)\right.$, where $r$ is a minimal usco retraction from $Y$ to $X$. It follows from Lemma 3(i) that there exists a countable $r$-admissible set $C$ containing $\bigcup\{B(U): U \in \mathscr{L}\}$. One can easily see that $p_{C}(x)=p_{C}(y)$ implies $f(x)=f(y)$ for every $x, y \in X$. Since $p_{C} \mid X$ is open, there exists a continuous function $g$ on $p_{C}(X)$ such that $f(x)=g\left(p_{C}(x)\right)$, for each $x \in X$. Since $p_{C}(X)$ is a usco retract of $Y_{C}$, it is closed in $Y_{C}$. Hence, $g$ is continuously extendable on $Y_{C}$; so $f$ is continuously extendable on $Y$.

(ii) Suppose $r$ is a minimal usco retraction from $Y$ to $X$. Let 2 be a family of standard open subsets of $Y$ such that card $\mathscr{Q}=w(X)$ and $\{U \cap X: U \in \mathscr{Q}\}$ is a base for $X$. Put $B_{1}=\bigcup\{m(U): U \in \mathscr{Q}\}$, where $m(U)=\left\{s \in S: p_{s}(U) \neq Y_{s}\right\}$. Clearly, card $B_{1}=w(X)$. By Lemma 3(i), pick a $r$-admissible set $B$ containing $B_{1}$ and such that card $B=w(X)$. Observe that $p_{B} \mid X$ is one-to-one. Since $p_{B} \mid X$ is open (by Lemma 6(i), we conclude that $p_{B} \mid X$ is a homeomorphism. Next, by Lemma 6(ii), $p_{B}(X)$ is a usco retract of $Y_{B}$.

THEOREM 1. For a space $X$, the following conditions are equivalent:

(i) $X \in \mathrm{AE}(0)$;

(ii) $X \in \mathrm{MAE}$;

(iii) $X$ is a usco retract of $R^{A}$, for some $A$.

Proof. (i) $\rightarrow$ (ii) Let $f: H \rightarrow X$ be a $Z$-normal mapping, where $H \subset Z$. Consider the absolute $a Z$ of $Z$ and the natural projection $g$ : $a Z \rightarrow Z$. Put $Y=g^{-1}(H)$. Observe that $f \circ g$ is $a Z$-normal. Since $\operatorname{dim} a Z$ $=0$ and $X \in \operatorname{AE}(0)$, there exists an extension $h: a Z \rightarrow X$ of $f \circ g$. Then the usco mapping $r: Z \rightarrow X$, defined by $r(z)=h\left(g^{-1}(z)\right)$, is an extension of $f$. Thus, $X \in \mathrm{MAE}$. 
(ii) $\rightarrow$ (iii) Denote by $C(X)$ the family of all continuous functions on $X$. Consider $X$ as a $C$-embedded subset of $R^{C(X)}$. Hence, there exists a usco retraction from $R^{C(X)}$ to $X$.

(iii) $\rightarrow$ (i) Let $\mathscr{K}$ be the class of all spaces $Y$ with the following property: $Y$ is a usco retract of $R^{A}$, for some $A$. We will prove (by transfinite induction) that every element of $\mathscr{K}$ is an $\mathrm{AE}(0)$-space. Let $X \in \mathscr{K}$ and $w(X)=\aleph_{0}$. In this case, by Lemma 7(ii), $X$ is a usco retract of $R^{\aleph_{0}}$. Hence, $X$ is a polish space and, by a result of Chigogidze [2], $X \in \mathrm{AE}(0)$. Assume that $\tau>\aleph_{0}$ and that for every $X \in \mathscr{K}$ with $w(X)<\tau$ we have $X \in \operatorname{AE}(0)$. Consider a space $X \in \mathscr{K}$ with $w(X)=\tau$. By Lemma 7(ii), $X$ is a usco retract of $R^{\tau}=\Pi\left\{R_{\alpha}: \alpha<\omega(\tau)\right\}$, where $\omega(\tau)$ is the initial ordinal of cardinality $\tau$. Let $r$ be a minimal usco retraction from $R^{\tau}$ to $X$. By Lemma 3(i), for every $\alpha<\omega(\tau)$ there exists a countable $r$-admissible set $B_{\alpha}$ containing $\alpha$. Next, denote $A(\alpha)=\bigcup\left\{B_{\beta}: \beta<\alpha\right\}$, $q_{\alpha}=p_{A(\alpha)} \mid X$ and $X_{\alpha}=q_{\alpha}(X)$ for each $\alpha<\omega(\tau)$. If $\alpha>\beta$ we put $p_{\beta}^{\alpha}=q_{\beta} \circ q_{\alpha}^{-1}$. Thus, we actually construct a continuous inverse system $S=\left\{X_{\alpha}, q_{\beta}^{\alpha}, \beta<\alpha<\Omega(\tau)\right\}$, in the sense of Ščepin [8], such that $X$ $=\lim _{\leftarrow} S$. According to Lemmas 3(ii) and 6, we have that, for every $\alpha<\omega(\tau), X_{\alpha} \in \mathscr{K}$ and $q_{\alpha}$ is functionally open. Hence, $q_{\alpha}^{\alpha+1}$ is functionally open. But $w\left(X_{\alpha}\right)<\tau$, so $X_{\alpha} \in \mathrm{AE}(0)$ for each $\alpha<\omega(\tau)$. Finally, Lemma 7(i) implies that $q_{\alpha}^{\alpha+1}$ has a polish kernel. Therefore, it follows from Lemma 5 that $q_{\alpha}^{\alpha+1}$ is 0 -soft for every $\alpha<\omega(\tau)$. So, all spaces $X_{\alpha}$ and all mappings $q_{\alpha}^{\alpha+1}$ are $\mathrm{AE}(0)$ and 0 -soft, respectively. Therefore, $X \in \mathrm{AE}(0)$.

LEMMA 8. Let $r$ be a usco mapping from $M$ to a compact space $X$ and let $M$ be a dense subset of $Y$. Then $r$ can be extended to a usco mapping from $Y$ to $X$.

Proof. For every $y \in Y$ denote by $U(y)$ the local base at $y$ in $Y$. Then the usco mapping $r_{1}$, defined by $r_{1}(y)=\bigcap\{\operatorname{cl}(r(U \cap M)): U \in$ $U(y)\}$, is the required extension.

LEMMA 9. Suppose $Z=\Pi\left\{Z_{s}: s \in S\right\}$ is a product of separable metric spaces and $Y$ is closed in $Z$. Let $r$ be a minimal usco mapping from $Z$ to $Y$ and let $X$ be a subset of $Y$ such that $r(x)=\{x\}$ for every $x \in X$. Then the following holds:

(i) $r(x)=\{x\}$ for every $x \in G_{\delta}(X)$;

(ii) $r\left(G_{\delta}(M)\right) \subset G_{\delta}(H)$ for every $H \subset Y$ and every $M \subset r^{\#}(H)$. 
Proof. (i) Suppose $r\left(x_{0}\right) \neq x_{0}$ for some $x_{0} \in G_{\delta}(x)$. Take a point $y \in r\left(x_{0}\right) \backslash\left\{x_{0}\right\}$ and a countable $r$-admissible set $B \subset S$ such that $p_{B}(y) \neq p_{B}\left(x_{0}\right)$. Since $p_{B}^{-1}\left(p_{B}\left(x_{0}\right)\right) \cap X \neq \varnothing$, choose $x \in p_{B}^{-1}\left(p_{B}\left(x_{0}\right)\right)$ $\cap X$. Lemma 4(ii) implies $p_{B}(x)=p_{B}\left(r\left(x_{0}\right)\right)$. This is impossible because $x_{0}, y \in r\left(x_{0}\right)$ and $p_{B}\left(x_{0}\right) \neq p_{B}(y)$. Hence, $r(x)=\{x\}$ for every $x \in$ $G_{\delta}(X)$.

(ii) Assume $H \subset Y$ and $M \subset r^{\#}(H)$. Let $r\left(x_{0}\right) \backslash G_{\delta}(H) \neq \varnothing$ for some $x_{0} \in G_{\delta}(M)$. Take a point $y \in r\left(x_{0}\right) \backslash G_{\delta}(H)$ and a countable $r$-admissible set $B \subset S$ such that $p_{B}(y) \notin p_{B}(H)$. Next choose a point $x \in p_{B}^{-1}\left(p_{B}\left(x_{0}\right)\right) \cap M$. Then, by Lemma 4(ii), we have $p_{B}(r(x))=$ $p_{B}\left(r\left(x_{0}\right)\right)$. But $r(x) \subset H$; so $p_{B}\left(r\left(x_{0}\right)\right) \subset p_{B}(H)$. This contradicts $p_{B}(y)$ $\notin p_{B}(H)$. Therefore, $r\left(G_{\delta}(M)\right) \subset G_{\delta}(H)$.

THEOREM 2. For a space $X$, the following conditions are equivalent:

(i) $X \in \operatorname{ANE}(0)$;

(ii) $X \in \mathrm{MANE}$;

(iii) $X$ is open in its Hewitt-realcompactification $\nu X$ and $\nu X \in \mathrm{AE}(0)$.

Proof. (i) $\rightarrow$ (ii) This implication can be proved as the implication (i) $\rightarrow$ (ii) of Theorem 1.

(ii) $\rightarrow$ (iii) Consider $X$ as a $C$-embedded subset of $R^{A}$, where $A$ is the family of all continuous functions on $X$. Clearly, $\nu X=\operatorname{cl}(X)$. Since $X \in$ MANE there exists a usco retraction $r_{1}$ from an open subset $U$ of $R^{A}$ to $X$. It is easily seen that $U \cap \nu X=X$ i.e. $X$ is open in $\nu X$. Identifying $R$ with $(0,1)$, we consider $R^{A}$ as a dense subset of $I^{A}$, where $I=[0,1]$. Put $Y=\mathrm{cl}_{I^{A}}(X)$. By Lemma 8 , there exists a usco extension $r_{2}$ : Int $I_{I^{A}}\left(\mathrm{cl}_{I^{A}}(U)\right) \rightarrow Y$ of $r_{1}$. Let $r_{3}$ be a usco mapping from $I^{A}$ to $Y$ defined by letting $r_{3}(y)=r_{2}(y)$, for $y \in \operatorname{Int}_{I^{A}}\left(\mathrm{cl}_{I^{A}}(U)\right)$, and $r_{3}(y)=Y$, otherwise. Denote by $r$ a minimal usco selection for $r_{3}$. Since each point $z \in I^{A} \backslash R^{A}$ is contained in a $G_{\delta}$-subset $H(z)$ of $I^{A}$ with $H(z) \cap R^{A}=\varnothing$, the $G_{\delta}$-closure $G_{\delta}(X)$ of $X$ in $I^{A}$ coincides with $\nu X$. So, by Lemma $9, r$ is a usco retraction from $G_{\delta}(U)$ to $\nu X$. Here, $G_{\delta}(U)$ is the $G_{\delta}$-closure of $U$ in $R^{A}$. It follows from Lemma 1 that there exists a countable set $B \subset A$ such that $G_{\delta}(U)=p_{B}(U) \times R^{A \backslash B}$. The space $p_{B}(U)$, being a polish space, is an $\mathrm{AE}(0)$. Hence, $G_{\delta}(U) \in \mathrm{AE}(0)$ as a product of $\mathrm{AE}(0)$-spaces. Thus, $\nu X$ is a usco retract of an $\mathrm{AE}(0)$-space. Therefore, by Theorem 1 , $\nu X \in \mathrm{AE}(0)$.

(iii) $\rightarrow$ (i) This implication is obvious. 
Corollary 1. Let $X \in \mathrm{A}(\mathrm{N}) \mathrm{E}(0)$ and let $F$ be $a G_{\delta}$-subset of $X$. Then the $G_{\delta}$-closure of $F$ in $X$ is also an $\mathrm{A}(\mathrm{N}) \mathrm{E}(0)$-space.

Proof. Let $X \in \mathrm{ANE}(0)$. Since $\nu X \in \mathrm{AE}(0)$ there is a minimal usco retraction $r$ from $R^{A}$ to $\nu X$ for some $A$. The set $F$ is $G_{\delta}$ in $\nu X$ because $X$ is open in $\nu X$. Hence, $r^{\#}(F)$ is a $G_{\delta}$-subset of $R^{A}$. By Lemma 1 , $G_{\delta}\left(r^{\#}(F)\right)$ is a product of polish spaces, so $G_{\delta}\left(r^{\#}(F)\right) \in \operatorname{AE}(0)$. Next, Lemma 9 implies that the $G_{\delta}$-closure $G_{\delta}(F)$ of $F$ in $\nu X$ is a usco retract of $G_{\delta}\left(r^{\#}(F)\right)$. Thus, $G_{\delta}(F)$ is also an $\mathrm{AE}(0)$-space. But $G_{\delta}(F) \cap X$ is open and dense in $G_{\delta}(F)$. Consequently $G_{\delta}(F) \cap X \in \operatorname{ANE}(0)$. However, $G_{\delta}(F) \cap X$ is the $G_{\delta}$-closure of $F$ in $X$.

By the same arguments one can prove that the $G_{\delta}$-closure of $F$ in $X$ is an $\mathrm{AE}(0)$-space if $X \in \mathrm{AE}(0)$.

THEOREM 3. Let $X$ be a pinnate in the sense of Arhangel'skii [1] $\mathrm{ANE}(0)$-space. Then $\nu X$ is Lindelöf and $\check{C}$ ech-complete.

Proof. First we will prove that $X$ is Čech-complete. Consider the Stone-Čech compactification $\beta X$ of $X$. Denote by $Z$ the space obtained from $\beta X$ by means of making the points of $\beta X \backslash X$ isolated. We observe that $X$ is a closed $C$-embedded subset of $Z$. Since $X \in \operatorname{ANE}(0)$, there is a usco retraction from $U$ to $X$, where $U$ is an open set in $Z$ containing $X$. Now, to prove that $X$ is Čech-complete one can use the arguments of Przymusinski [7, the proof of Lemma 2].

Next, let $r_{1}$ be a usco mapping from $R^{A}$ to $\nu X$ for some $A$. Consider $R^{A}$ as a dense subset of $I^{A}$ by identifying $R$ with $(0,1)$, and put $Y=\mathrm{cl}_{I^{A}}(\nu X)$. By Lemma 8, $r_{1}$ is extendable to a usco mapping $r$ from $I^{A}$ to $Y$. Wlog, we assume that $r$ is minimal. Put $H=r^{\#}(X) . H$ is a $G_{\delta}$-subset of $I^{A}$ because $X$ is Čech-complete. Since $G_{\delta}(X)=\nu X$, it follows from Lemma 9 that $r$ is a usco retraction from $G_{\delta}(H)$ to $\nu X$. So, $\nu X$ is closed in $G_{\delta}(H)$. But, by Lemma $1, G_{\delta}(H)$ is a Lindelöf $G_{\delta}$-subset of $I^{A}$. Therefore, $\nu X$ is Lindelöf and Čech-complete.

COROllary 2. Every pinnate $\mathrm{AE}(0)$-space is Lindelöf and $\check{C}$ ech-complete.

An embedding $j$ of $X$ in $Y$ is said to be $d$-regular [11] (br. a $d$-embedding) if for every open subset $U$ of $j(X)$ there exists an open subset $e(U)$ of $Y$ such that the following conditions are fulfilled:

(1) $e(\varnothing)=\varnothing$;

(2) $e(U) \cap j(X)=U$;

(3) $e(U) \cap e(V)=e(U \cap V)$; 
Shirokov [11] proved that $X$ is a Dugundji space if and only if every embedding of $X$ in a Tychonoff cube is a $d$-embedding. We give a similar characterization of Čech-complete $\mathrm{AE}(0)$-spaces.

THEOREM 4. For a Čech-complete space $X$ the following conditions are equivalent:

(i) $\nu X$ is a Čech-complete Lindelöf $\mathrm{AE}(0)$-space;

(ii) every $C$-embedding of $X$ in any space is a d-embedding;

(iii) $X$ is a d-embedded subset of $R^{A}$, for some $A$.

Proof. (i) $\rightarrow$ (ii) Suppose $X$ is a $C$-embedded subsert of a space $Y$. Then there exists a mapping $h: Y \rightarrow R^{C(X)}$ such that $h \mid X$ is a homeomorphism and $\mathrm{cl}_{R^{C(X)}}(h(X))=\nu X$. Let $r$ be a usco retraction from $R^{C(X)}$ to $v X$. For every open set $U$ in $X$, we let $e(U)=h^{-1}\left(r^{\#}(V(U))\right)$, where $V(U)=\bigcup\{W: W$ is open in $\nu X$ and $W \cap h(X)=h(U)\}$. It is easily seen that this operator satisfies the above three conditions. Thus, $X$ is $d$-embedded in $Y$.

(ii) $\rightarrow$ (iii) This implication is obvious.

(iii) $\rightarrow$ (i) Let $X$ be a $d$-embedded subset of $R^{A}$ for some $A$. So, there exists a $d$-regular operator $e$ from the topology of $X$ to the topology of $R^{A}$. Consider $R^{A}$ as a dense subset of $I^{A}$ and put $Y=\mathrm{cl}_{I^{A}}(X)$. Define a usco mapping $r_{1}$ from $R^{A}$ to $Y$ by letting $r_{1}(x)=\bigcap\left\{\operatorname{cl}_{Y}(U): x \in e(U)\right\}$, for $x \in \bigcup\{e(U): U$ is open in $X\}$, and $r_{1}(x)=Y$, otherwise. Clearly, $r_{1}(x)=\{x\}$ for every $x \in X$. Next, by Lemma $8, r_{1}$ is extendable to a usco mapping $r$ from $I^{A}$ to $Y$. We assume that $r$ is minimal. Since $X$ is Čech-complete, the set $H=r^{\#}(X)$ is $G_{\delta}$ in $I^{A}$. Lemma 9 implies that $r$ is a usco retraction from $G_{\delta}(H)$ to $G_{\delta}(X)$. By Lemma $1, G_{\delta}(H)$ is a Lindelöf Čech-complete AE(0)-space. Therefore, $G_{\delta}(X)$ being a usco retract of $G_{\delta}(H)$, is a Lindelöf Čech-complete $\mathrm{AE}(0)$-space too. It remains to prove that $G_{\delta}(X)$ is the Hewitt-realcompactification of $X$. It is known [2] that every $\mathrm{AE}(0)$-space is perfectly $k$-normal in the space of Šcepin [10] and that every $G_{\delta}$-dense subset of a perfectly $k$-normal space $Z$ is $C$-embedded in $Z$ [12]. Hence, $X$ is $C$-embedded in $G_{\delta}(X)$. Therefore, $G_{\delta}(X)$ is the Hewitt-realcompactification of $X$.

COROLlaRy 3. For a Čech-complete realcompact space $X$ the following conditions are equivalent:

(i) $X$ is a Lindelöf $\mathrm{AE}(0)$-space;

(ii) every $C$-embedding of $X$ in any space is a d-embedding;

(iii) $X$ is a d-embedded subset of $R^{A}$, for some $A$. 
Let us note that the completeness in Theorem and Corollary 3 is essential. Indeed, every non-complete subspace of $R^{\aleph_{0}}$ is $d$-embedded in $R^{\aleph_{0}}$ but is not an $\mathrm{AE}(0)$-space.

We have been unable to decide the following problems: Is every Lindelöf $\mathrm{AE}(0)$-space Čech-complete? Is every normal $\mathrm{AE}(0)$-space Lindelöf?

\section{REFERENCES}

[1] A. V. Arhangel'skii, On a class of spaces containing all metric spaces and all locally compact space, Amer. Math. Soc. Transl., 92 (1970), 1-39.

[2] A. Chigogidze, Nesčetnye stepeni prjamoi, natural'novo rjada $i$ n-mjagkie otobrajenija, Dokl. Akad. Nauk SSSR, 278 (1984), 50-53.

[3] A. N. Dranishnikov, Mnogoznačnye absolutnye retracti $i$ absolutnye ekstenzory $v$ razmernostjah 0 i 1, Usp. Matem. Nauk, 39:5 (1984), 241-242.

[4] R. Haydon, On a problem of Pelczynski: Milutin spaces, Dugundji spaces and AE(dim 0), Studia Math., 52 (1974), 23-31.

[5] A. Pelčzynski, Linear extensions, linear averagings, and their applications to linear classification of spaces of continuous functions, Warszawa 1968.

[6] R. Pol and E. Pol, Remarks on Cartesian products, Fund. Math., 93 (1976), 57-69.

[7] T. Przymusinski, Colectionwise normality and absolute retracts, Fund. Math., 98 (1978), 61-73.

[8] E. Ščepin, Topologija predel'nyh prostranstv nesčetnyh obratnyh spektrov, Usp. Matem. Nauk, 31:5 (1976), 191-226.

[9] _ Funktory $i$ nesčetnye stepeni kompaktov, Usp. Matem. Nauk, 36:3 (1981), $3-62$.

[10] _ O topologičeskih proizvedenijah, gruppah $i$ novom klasse prostranstv bolee občih čem metričeskie, Dokl. Akad. Nauk SSSR, 226 (1976), 527-529.

[11] L. Shirokov, Vneshnaja harakteristika prostranstv Dugundji $i$ k-metrizuemyh prostranstv, Dokl. Akad. Nauk SSSR, 263 (1982), 1073-1077.

[12] M. Tkačenko, The notion of o-tightness and C-embedded subspaces of products, Topology Appl., 15 (1983), 93-98.

Received August 13, 1985 and in revised form April 16, 1986.

1090 SIFIA

P. O. Box 373

Bulgaria 


\section{PACIFIC JOURNAL OF MATHEMATICS EDITORS}

\author{
V. S. VARADARAJAN \\ (Managing Editor) \\ University of California \\ Los Angeles, CA 90024 \\ Herbert Clemens \\ University of Utah \\ Salt Lake City, UT 84112 \\ R. FINN \\ Stanford University \\ Stanford, CA 94305
}

\author{
HERMANN FLASCHKA \\ University of Arizona \\ Tucson, AZ 85721 \\ RAMESh A. GANGOLli \\ University of Washington \\ Seattle, WA 98195 \\ VAUGHAN F. R. JONES \\ University of California \\ Berkeley, CA 94720 \\ ROBION KIRBY \\ University of California \\ Berkeley, CA 94720 \\ C. C. MOORE \\ University of California \\ Berkeley, CA 94720 \\ H. SAMELSON \\ Stanford University \\ Stanford, CA 94305 \\ HAROLD STARK \\ University of California, San Diego \\ La Jolla, CA 92093
}

\section{ASSOCIATE EDITORS}
R. ARENS
E. F. BECKENBACH
B. H. NEUMANN
F. WOLF
K. YOSHIDA (1906-1982)

\section{SUPPORTING INSTITUTIONS}
UNIVERSITY OF ARIZONA
UNIVERSITY OF BRITISH COLUMBIA
CALIFORNIA INSTITUTE OF TECHNOLOGY
UNIVERSITY OF CALIFORNIA
MONTANA STATE UNIVERSITY
UNIVERSITY OF NEVADA, RENO
NEW MEXICO STATE UNIVERSITY
OREGON STATE UNIVERSITY
UNIVERSITY OF OREGON UNIVERSITY OF SOUTHERN CALIFORNIA STANFORD UNIVERSITY UNIVERSITY OF HAWAII UNIVERSITY OF TOKYO UNIVERSITY OF UTAH WASHINGTON STATE UNIVERSITY UNIVERSITY OF WASHINGTON 


\section{Pacific Journal of Mathematics \\ Vol. 127, No. $1 \quad$ January, 1987}

Jacob Burbea, Boundary behavior of holomorphic functions in the ball ..... 1

Jan Dijkstra, Strong negligibility of $\sigma$-compacta does not characterize

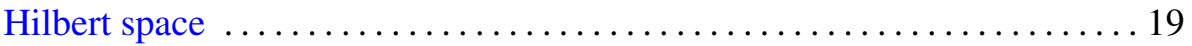

Ruy Exel, Rotation numbers for automorphisms of $C^{*}$ algebras $\ldots \ldots \ldots 31$

Howard Jacobowitz, The canonical bundle and realizable CR

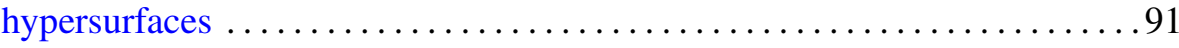

James T. Joichi and Dennis Warren Stanton, Bijective proofs of basic

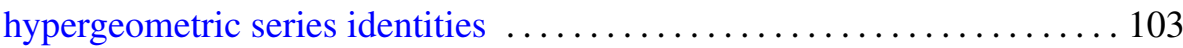

Gareth J. Knowles, Quotients of nest algebras with trivial commutator .... 121

Murray Angus Marshall, Exponentials and logarithms on Witt rings ..... 127

Courtney Hughes Moen, The dual pair $(U(3), U(1))$ over a $p$-adic field . ...141

William Ortmeyer, Surgery on a class of pretzel knots $\ldots \ldots \ldots \ldots \ldots \ldots 155$

John Gerard Ryan, Extensions of representations of Lie algebras . . . . . 173

Ivan Charles Sterling, A generalization of a theorem of Delaunay to

rotational $W$-hypersurfaces of $\sigma_{l}$-type in $H^{n+1}$ and $S^{n+1} \ldots \ldots \ldots 187$

Vesko M. Valov, Another characterization of AE(0)-spaces $\ldots \ldots \ldots \ldots \ldots 9$ 